\title{
Professions à risque pour l'acquisition des zoonoses d'importance pour la santé publique au Québec
}

\author{
Ariane Adam-Poupart 1,2,3*, Laurie-Maude Drapeau ${ }^{1}$, Sadjia Bekal ${ }^{4}$, Geneviève Germain ${ }^{1,5}$, \\ Alejandra Irace-Cima ${ }^{1,2}$, Marie-Pascale Sassine ${ }^{1}$, Audrey Simon ${ }^{3,5}$, Julio Soto ${ }^{1,2}$, Karine Thivierge ${ }^{4,6}$, \\ France Tissot $^{1}$
}

\section{Résumé}

Contexte : Les changements climatiques jouent un rôle important dans l'établissement et l'expansion géographiques des zoonoses. Il est donc essentiel de connaître les populations à risque de contracter ces maladies pour ensuite orienter les politiques et les pratiques en santé publique. Au Québec, 14 zoonoses ont été identifiées importantes pour la santé publique afin de guider les efforts d'adaptation aux changements climatiques des décideurs et des chercheurs. Cette étude vise donc à dresser un portrait des professions et des secteurs d'activité économique à risque pour ces zoonoses.

Méthode : Un examen rapide de la littérature scientifique a été réalisé. Des recherches ont été effectuées dans les bases de données des plateformes de recherche Ovid et EBSCO afin de repérer des articles publiés entre 1995 et 2018, en anglais et en français, sur 14 zoonoses (campylobactériose, cryptosporidiose, Escherichia coli vérocytotoxinogène, giardiase, listériose, salmonellose, encéphalite équine de l'Est, maladie de Lyme, virus du Nil occidental, botulisme $d^{\prime}$ 'origine alimentaire, fièvre $Q$, grippe aviaire et porcine, rage, syndrome pulmonaire à hantavirus) et la santé au travail. La recherche documentaire a permis de repérer 12558 articles et, après élimination des doublons, 6838 articles ont été évalués en fonction du titre et du résumé. Les articles admissibles devaient aborder les deux concepts de base de la recherche (zoonoses priorisées et santé des travailleurs). Parmi les 621 articles jugés admissibles, 110 ont été retenus à la suite de leur lecture intégrale.

Résultats : Parmi les zoonoses à l'étude, les zoonoses entériques ont été les plus fréquemment rapportées. L'agriculture, qui inclut les services vétérinaires, les services de l'administration publique, de même que les services médicaux et sociaux, ont été les secteurs les plus fréquemment identifiés dans la littérature.

Conclusion : Les résultats de notre étude aideront les autorités de la santé publique et les décideurs à cibler les secteurs et les professions qui sont particulièrement à risque pour l'acquisition de zoonoses. Ils permettront également d'optimiser les pratiques de santé publique des acteurs responsables de la santé des travailleurs.
Cette oeuvre est mise à la disposition selon les termes de la licence internationale Creative Commons Attribution 4.0

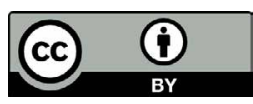

Affiliations

1 Direction des risques biologiques et de la santé au travail, Institut national de santé publique du Québec, Québec, QC

2 École de santé publique de I'Université de Montréal (ESPUM), Montréal, QC

${ }^{3}$ Groupe de recherche en épidémiologie des zoonoses et santé publique (GREZOSP), Faculté de médecine vétérinaire (FVM), Université de Montréal, Montréal, QC

${ }^{4}$ Laboratoire de santé publique du Québec, Institut national de santé publique du Québec, Sainte-Anne-de-Bellevue, QC

${ }^{5}$ Observatoire multipartite québécois sur les zoonoses et I'adaptation aux changements climatiques du Québec

${ }^{6}$ Institut de parasitologie, Faculté des sciences de l'agriculture et de l'environnement, Université McGill, Sainte-Anne-de-Bellevue, QC

\section{${ }^{\star}$ Correspondance :}

ariane.adam-poupart@inspq.qc.ca

Citation proposée : Adam-Poupart A, Drapeau LM, Bekal S, Germain G, Irace-Cima A, Sassine MP, Simon A

Soto J, Thivierge K, Tissot F. Professions à risque pour l'acquisition des zoonoses d'importance pour la santé publique au Québec. Relevé des maladies transmissibles au Canada 2021;47(1):56-67.

https://doi.org/10.14745/ccdr.v47i01a08f

Mots-clés : zoonoses, professions, secteurs d'activité, travailleurs, santé publique

\section{Introduction}

Les changements climatiques jouent un rôle important dans l'établissement géographique et la propagation des zoonoses. Les variations prévues de la température et des précipitations influeront sur la survie et la propagation des pathogènes zoonotiques, ainsi que sur la répartition de leurs vecteurs, favorisant ainsi la dispersion spatio-temporelle de ces maladies (1). 
Au Québec, 14 zoonoses ont été identifiées comme importantes pour la santé publique. II s'agit des douze zoonoses priorisées par l'Observatoire multipartite québécois sur les zoonoses et l'adaptation aux changements climatiques, un organisme composé d'experts scientifiques et de représentants des décideurs de politiques publiques, auxquelles se sont ajoutées la listériose et le syndrome pulmonaire à hantavirus (1-3). Ces 14 zoonoses sont entériques (campylobactériose, cryptosporidiose, Escherichia coli vérocytotoxinogène, giardiase, listériose, salmonellose) et non entériques (transmission vectorielle, soit encéphalite équine de l'Est, maladie de Lyme, virus du Nil occidental et transmission non vectorielle, soit botulisme alimentaire au Nunavik, fièvre $Q$, grippe aviaire et porcine, rage, syndrome pulmonaire à hantavirus). L'Observatoire a publié de l'information sur les populations vulnérables à ces maladies, y compris les chasseurs de phoques du Nunavik, qui risquent de contracter le botulisme d'origine alimentaire, et les travailleurs de l'industrie avicole, qui risquent de contracter la campylobactériose $(2,3)$. L'information ciblant les travailleurs demeure toutefois incomplète ou même inexistante pour certaines zoonoses, ce qui indique la nécessité de développer ce corpus de connaissances pour orienter les politiques et pratiques en matière de santé publique.
Cette étude visait à déterminer les professions et secteurs d'activité économique les plus à risque pour l'acquisition de zoonoses importantes pour la santé publique au Québec pour ainsi contribuer à la prise de décisions des autorités de santé publique et d'optimiser les pratiques des acteurs responsables de la santé des travailleurs. Cette synthèse des connaissances tirées de la littérature scientifique est présentée par catégorie de zoonoses (entériques, non entériques vectorielles et non entériques non vectorielles).

\section{Méthode}

L'équipe de recherche a réalisé une revue rapide de la littérature reposant sur une méthodologie de revue systématique. Les plateformes Ovid et EBSCO ont été utilisées pour effectuer des recherches dans les bases de données Medline, Embase, Evidence-Based Medicine Reviews (EBMR), Global Health, Forfait Total Access Collection et Environment Complete. Ces recherches ont été effectuées à l'aide d'une série de mots-clés associés aux zoonoses concernées et à la santé des travailleurs. Les tableau 1 ainsi que le tableau 2 présentent les requêtes élaborées à l'aide de ces mots-clés.

\section{Tableau 1 : Requêtes dans les bases de données Ovid}

\begin{tabular}{|c|c|}
\hline $\begin{array}{l}\text { Nombre de } \\
\text { recherches }\end{array}$ & Requêtes \\
\hline S1 & 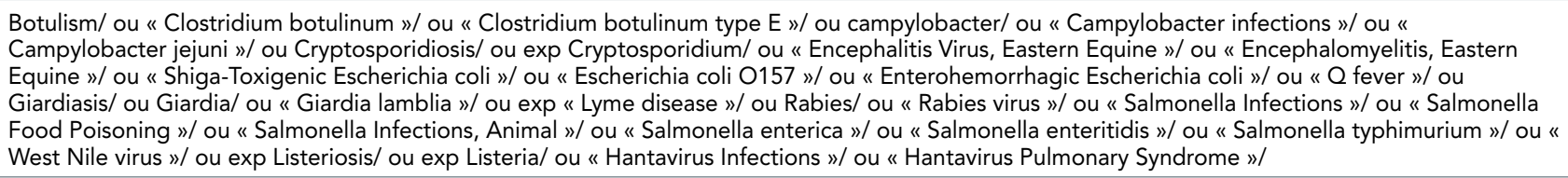 \\
\hline $\mathrm{S} 2$ & $\begin{array}{l}\text { ("Influenza A virus »/ ou " Influenza A Virus, H1N1 Subtype »/ ou " Influenza A Virus, H1N2 Subtype »/ ou " Influenza A Virus, H3N2 Subtype »/ ou « } \\
\text { Influenza A Virus, H5N1 Subtype »/ ou « Influenza A Virus, H7N9 Subtype »/ ou « Influenza in Birds »/) et Zoonoses/ }\end{array}$ \\
\hline S3 & 1 ou 2 \\
\hline S4 & 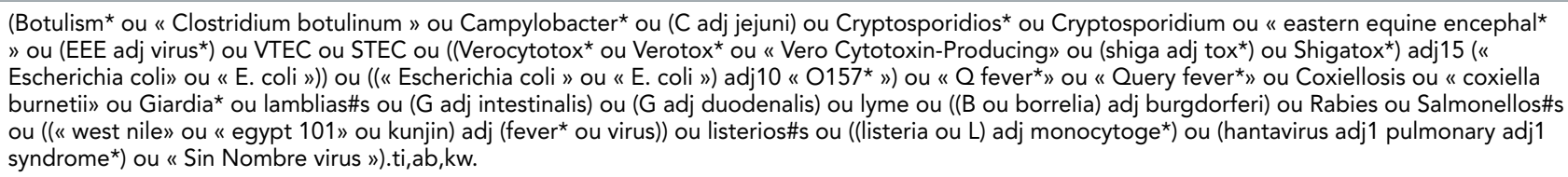 \\
\hline S5 & 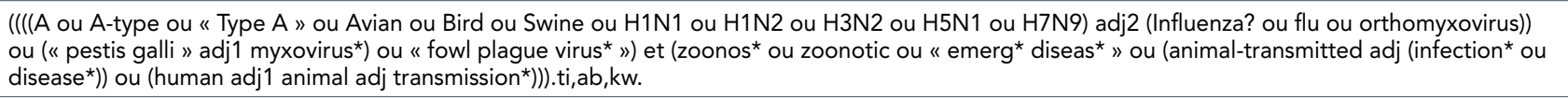 \\
\hline S6 & 4 ou 5 \\
\hline S7 & 3 ou 6 \\
\hline S8 & $\begin{array}{l}\text { * } \text { occupational exposure »/ ou * « occupational health »/ ou exp * « occupational groups »/ ou * } \text { " occupational diseases »/ ou * } \text { " agricultural workers' } \\
\text { diseases »/ ou « meat-packing industry »/ }\end{array}$ \\
\hline S9 & (occupation* ou worker* ou workplace* ou professional* ou employ* ou job\$1 ou labo?r ou labo?rs ou labo?rer* ou personnel ou staff).ti,ab,kw. \\
\hline S10 & (farm* ou agricultur* ou hunter* ou (outdoor adj occupation*) ou veterinar* ou (wildlife adj manag*) ou abattoir* ou slaughter*).ti,ab,kw. \\
\hline S11 & 8 ou 9 ou 10 \\
\hline S12 & 7 et 11 \\
\hline S13 & 12 pas (exp animals/ not humans/) \\
\hline S14 & 13 et (anglais ou français).lg. \\
\hline S15 & Limite 14 à l'année 1995-2018 \\
\hline S16 & 15 non (editorial ou letter ou comment ou news).pt. \\
\hline
\end{tabular}


Tableau 2 : Requêtes dans la base de données EBSCO OU « eastern equine encephal* " OU (EEE WO virus*) OU VTEC OU STEC OU ((Verocytotox* ou Verotox* ou " Vero CytotoxinProducing » ou (shiga w0 tox*) OU Shigatox») W15 ("Escherichia coli » » ou «E. coli »)) OU (( Escherichia coli » ou «E. coli ») W10 « O157* ») OU « Q fever* » OU « Query fever* » OU Coxiellosis OU « coxiella burnetii » OU Giardia* OU lamblias\#s OU (G W0 intestinalis) OU (G W0 duodenalis) OU lyme ou ((B ou borrelia) W0 burgdorferi) OU Rabies OU Salmonellos\#s OU ((« west nile » OU « egypt 101 » OU kunjin) W0 (fever* OU virus)) OU listerios\#s OU ((listeria OU L) W0 monocytoge*) OU (hantavirus W1 pulmonary W1 syndrome*) OU "Sin Nombre virus ») OU AB (Botulism* OU « Clostridium botulinum » OU Campylobacter* OU (C W0 jejuni) OU Cryptosporidios* OU Cryptosporidium OU « eastern equine encephal* » OU (EEE WO virus*) OU VTEC OU STEC OU ((Verocytotox* ou Verotox* ou « Vero Cytotoxin-Producing » ou (shiga W0 tox*) OU Shigatox*) W15 (" Escherichia coli » ou " E. coli »)) OU ((« Escherichia coli » ou «E. coli ») W10 «O157*») OU « Q fever* » OU « Query fever* » OU Coxiellosis OU « coxiella burnetii » OU Giardia* OU lamblias\#s OU (G W0 intestinalis) OU (G W0 duodenalis) OU lyme ou ((B ou borrelia) W0 burgdorferi) OU Rabies OU Salmonellos\#s OU ((« west nile » OU « egypt 101 » OU kunjin) W0 (fever* OU virus)) OU listerios\#s OU ((listeria OU L) W0 monocytoge*) OU (hantavirus W1 pulmonary W1 syndrome*) OU « Sin Nombre virus ») OU KW (Botulism* OU « Clostridium botulinum » OU Campylobacter* OU (C WO jejuni) OU Cryptosporidios* OU Cryptosporidium OU " eastern equine encephal* » OU (EEE WO virus*) OU VTEC OU STEC OU ((Verocytotox* ou Verotox* ou "Vero Cytotoxin-Producing " ou (shiga WO tox*) OU Shigatox*) W15 (« Escherichia coli » ou «E. coli »)) OU ((« Escherichia coli » ou « E. coli ») W10 « O157* ») OU «Q fever* » OU « Query fever* » OU Coxiellosis OU « coxiella burnetii » OU Giardia* OU lamblias\#s OU (G WO intestinalis) OU (G WO duodenalis) OU lyme ou ((B ou borrelia) W0 burgdorferi) OU Rabies OU Salmonellos\#s OU ((« west nile » OU « egypt 101 » OU kunjin) W0 (fever* OU virus)) OU listerios\#s OU ((listeria OU L) W0 monocytoge*) OU (hantavirus W1 pulmonary W1 syndrome*) OU « Sin Nombre virus $»)$

S2 $\quad$ TI (((A OU A-type OU « Type A » OU Avian OU Bird OU Swine OU H1N1 OU H1N2 OU H3N2 OU H5N1 OU H7N9) W2 (Influenza\# OU flu OU orthomyxovirus)) OU (« pestis galli » W1 myxovirus*) OU « fowl plague virus* ») ET (zoonos* OU zoonotic OU « emerg* diseas* » OU (animal-transmitted W0 (infection* OU disease*)) OU (human W1 animal W0 transmission*))) OU AB ((((A OU A-type OU « Type A » OU Avian OU Bird OU Swine OU H1N1 OU H1N2 OU H3N2 OU H5N1 OU H7N9) W2 (Influenza\# OU flu OU orthomyxovirus)) OU (« pestis galli » W1 myxovirus*) OU « fowl plague virus* ») ET (zoonos* OU zoonotic OU « emerg* diseas* » OU (animal-transmitted WO (infection* OU disease*)) OU (human W1 animal W0 transmission*))) OU KW ((((A OU A-type OU « Type A » OU Avian OU Bird OU Swine OU H1N1 OU H1N2 OU H3N2 OU H5N1 OU H7N9) W2 (Influenza\# OU flu OU orthomyxovirus)) OU (" pestis galli » W1 myxovirus*) OU « fowl plague virus* ») ET (zoonos* OU zoonotic OU " emerg* diseas* » OU (animaltransmitted W0 (infection* OU disease*)) OU (human W1 animal W0 transmission*)))

\begin{tabular}{|c|c|}
\hline S3 & S1 OU S2 \\
\hline S4 & 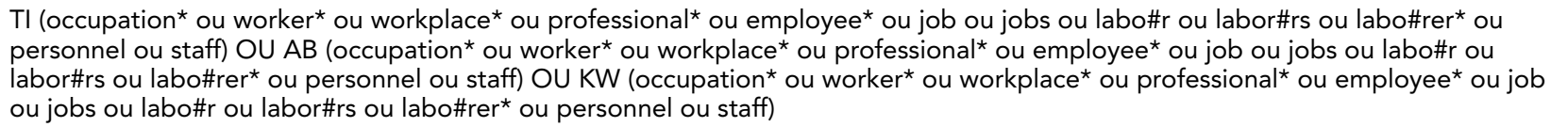 \\
\hline S5 & 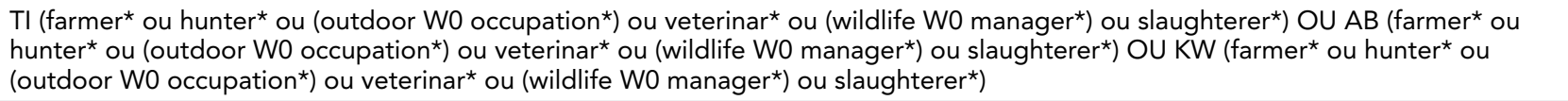 \\
\hline S6 & S4 OU S5 \\
\hline S7 & S3 ET S6 \\
\hline S8 & S7 et LA (anglais OU français) \\
\hline S9 & S8 et (DT 1995-2018) \\
\hline $\mathrm{S} 10$ & S9 NON PT (editorial ou letter ou commentary) \\
\hline S11 & 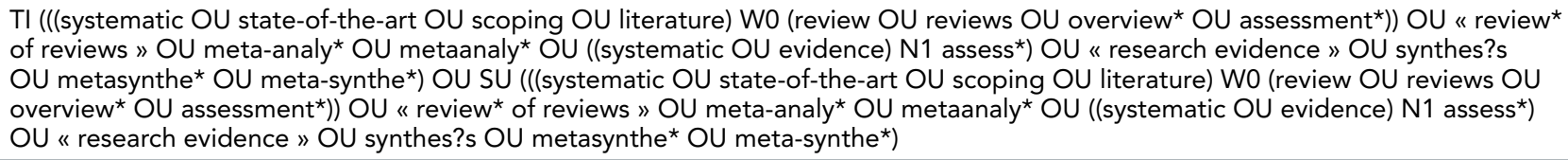 \\
\hline $\mathrm{S} 12$ & S10 ET S11 \\
\hline $\mathrm{S} 13$ & S10 ET NON S11 \\
\hline
\end{tabular}

La recherche s'est limitée aux études originales évaluées par des pairs et publiées entre 1995 et 2018 , en anglais ou en français. Les analyses documentaires, les commentaires, les éditoriaux, les nouvelles, les lettres d'opinion et les questions-réponses ont été exclus. Aucune restriction n'a été appliquée en termes de portée géographique. Le tri des articles a tout d'abord été fait sur la base des titres et des résumés. Les articles admissibles devaient démontrer un lien clair avec la recherche, c'est-à-dire aborder les deux concepts de la question de recherche (zoonoses prioritaires et santé des travailleurs) et traiter au minimum d'un secteur d'activité économique ou d'une profession à haut risque. Ensuite, une lecture complète des publications sélectionnées a conduit à ne retenir que les articles traitant spécifiquement des zoonoses visées et faisant référence au lieu de travail comme lieu $d$ 'acquisition. Les études ne portant pas sur un environnement de travail (i.e. une acquisition communautaire) ou mentionnant 
des circonstances d'exposition ne pouvant survenir dans les milieux de travail québécois ont été exclues. L'examen des références énumérées dans les publications sélectionnées a permis d'identifier des éléments pertinents dans un article publié avant 1995. Enfin, les études pour lesquelles les descriptions des cas de zoonoses ne répondaient pas aux critères des définitions nosologiques provinciales ou aux critères de diagnostic utilisés au Québec ont également été exclues. Les données recueillies à partir des articles sélectionnés (secteurs d'activité économique, professions à risque, facteurs de risque) ont été compilées et résumées dans des grilles d'analyse sommaires.

La figure 1 montre le processus qui a mené à la sélection des articles. L'équipe de recherche a déterminé les professions et les secteurs d'activité économique les plus à risque pour I'acquisition de ces zoonoses en se basant sur le nombre d'articles portant sur chacun.

\section{Résultats}

Le tableau 3 présente une liste des professions et des secteurs d'activité économique les plus à risque pour l'acquisition de zoonoses prioritaires. Ils sont classés en fonction de la Classification nationale des professions (2016 version 1.3) et la Classification des activités économiques du Québec 1984, version 1990. La répartition des articles sélectionnés par zoonose prioritaire est présentée au tableau 4.
Figure 1 : Illustration du processus de recherche de documentation et de sélection des publications Algorithmes de recherche dans les bases de données

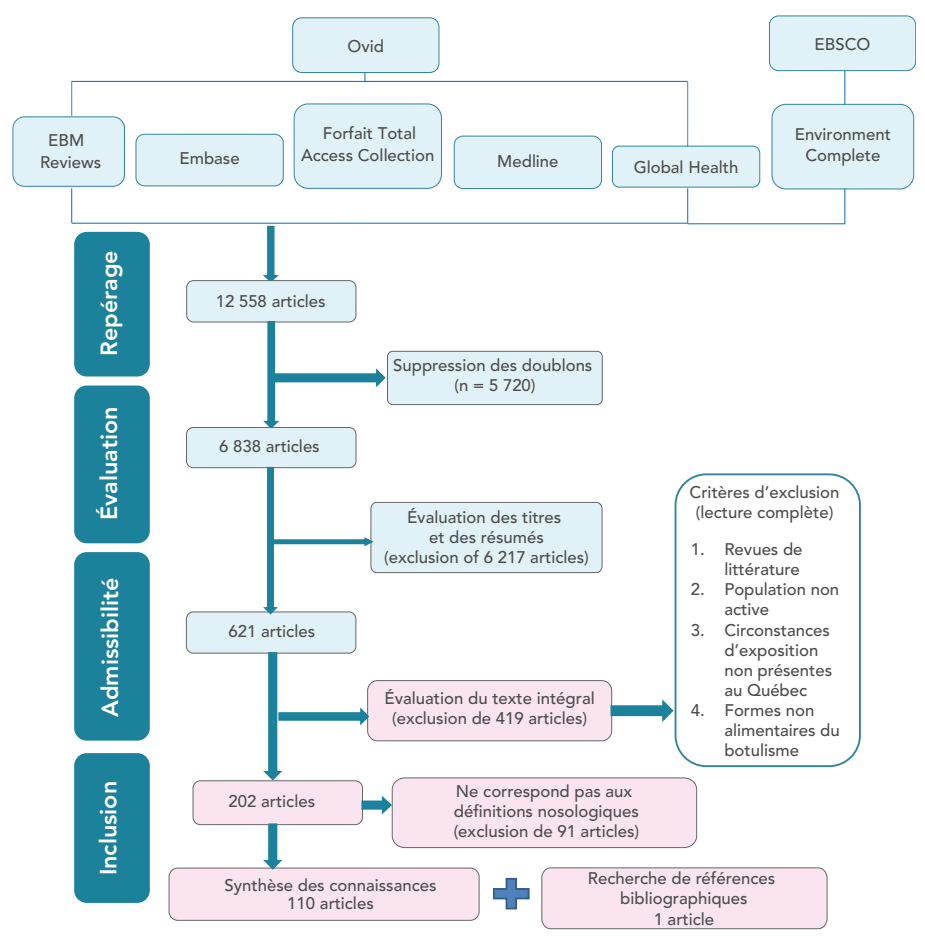

Remarque : Cinq bases de données ont été interrogées sur la plateforme Ovid, soit Embase, Ovid MEDLINE, Evidence-Based Medicine Reviews (EBMR), Forfait Total Access Collection et Global Health. Une base de données a été interrogée sur la plateforme EBSCO, soit Environment Complete (EC)

Restrictions utilisées : de 1995 à 2018; anglais et français; commentaires, éditoriaux, nouvelles, lettres d'opinion et questions-réponses exclus. Aucune restriction en termes de portée géographique n'a été appliquée pour lancer la recherche

Tableau 3 : Les catégories de zoonoses, leurs principaux réservoirs animaux au Québec et les principaux secteurs d'activité économique et professions indiqués comme étant à risque pour l'acquisition de ces zoonoses dans la littérature scientifique

\begin{tabular}{|c|c|c|c|}
\hline Zoonoses & $\begin{array}{l}\text { Principaux } \\
\text { réservoirs animaux }\end{array}$ & $\begin{array}{l}\text { Principaux secteurs d'activité } \\
\text { économique }\end{array}$ & Professions et références \\
\hline \multicolumn{4}{|l|}{ Zoonoses entériques } \\
\hline \multirow{2}{*}{ Campylobactériose } & \multirow{2}{*}{ Volaille } & Agriculture & Travailleurs agricoles, travailleurs de l'industrie avicole (4-13) \\
\hline & & Administration publique & Personnel militaire (14-19) \\
\hline \multirow{3}{*}{ Cryptosporidiose } & \multirow{3}{*}{$\begin{array}{l}\text { Bovins et autres } \\
\text { ruminants }\end{array}$} & Agriculture & $\begin{array}{l}\text { Étudiants en médecine vétérinaire (20-27), ouvriers agricoles } \\
\text { (28-33) et intervenants agricoles d'urgence }(34,35)\end{array}$ \\
\hline & & $\begin{array}{l}\text { Autres services commerciaux et } \\
\text { personnels }\end{array}$ & $\begin{array}{l}\text { Accompagnateurs d'excursions et employés de camps de } \\
\text { vacances (36-38) }\end{array}$ \\
\hline & & Services médicaux et sociaux & $\begin{array}{l}\text { Personnel des services de garde (39) et personnel des } \\
\text { laboratoires de recherche sur les animaux (40) }\end{array}$ \\
\hline \multirow{4}{*}{$\begin{array}{l}\text { Escherichia coli } \\
\text { vérocytotoxinogène }\end{array}$} & \multirow{4}{*}{$\begin{array}{l}\text { Bovins, autres ruminants } \\
\text { ou mammifères } \\
\text { herbivores }\end{array}$} & Agriculture & Travailleurs agricoles $(41-48)$ \\
\hline & & Services médicaux et sociaux & $\begin{array}{l}\text { Personnel des services de garde }(49,50) \text {, personnel hospitalier } \\
\text { (infirmières) et personnel œuvrant dans les maisons de soins } \\
\text { infirmiers }(51,52)\end{array}$ \\
\hline & & Enseignement et services annexes & $\begin{array}{l}\text { Employés en milieu scolaire (enseignants et aides-éducateurs) } \\
\text { (53) }\end{array}$ \\
\hline & & Administration publique & Personnel militaire (54) \\
\hline Giardiase & $\begin{array}{l}\text { Bovins, mammifères } \\
\text { sauvages }\end{array}$ & Services médicaux et sociaux & Personnel des services de garde (55-57) \\
\hline
\end{tabular}


Tableau 3 : Les catégories de zoonoses, leurs principaux réservoirs animaux au Québec et les principaux secteurs d'activité économique et professions indiqués comme étant à risque pour l'acquisition de ces zoonoses dans la littérature scientifique (suite)

\begin{tabular}{|c|c|c|c|}
\hline Zoonoses & $\begin{array}{l}\text { Principaux } \\
\text { réservoirs animaux }\end{array}$ & $\begin{array}{l}\text { Principaux secteurs d'activité } \\
\text { économique }\end{array}$ & Professions et références \\
\hline \multicolumn{4}{|c|}{ Zoonoses entériques (suite) } \\
\hline Listériose & $\begin{array}{l}\text { Bovins, ovins, porcs, } \\
\text { chèvres }\end{array}$ & Agriculture & Vétérinaires $(58,59)$ et ouvriers agricoles $(60)$ \\
\hline \multirow{7}{*}{ Salmonellose } & \multirow{7}{*}{ Volailles, porcs, bovins } & Agriculture & $\begin{array}{l}\text { Techniciens et professionnels de la médecine vétérinaire } \\
\text { (61-64), ouvriers agricoles (65-67), employés des élevages de } \\
\text { serpents (68) }\end{array}$ \\
\hline & & Services médicaux et sociaux & $\begin{array}{l}\text { Travailleurs de la santé (69-71), personnel dans les maisons de } \\
\text { retraite }(72) \text { et personnel des services de garde }(73,74)\end{array}$ \\
\hline & & Administration publique & Personnel militaire $(75,76)$ \\
\hline & & $\begin{array}{l}\text { Industries manufacturières } \\
\text { diverses }\end{array}$ & Personnel de l'industrie des animaux de compagnie (77) \\
\hline & & Industrie des aliments et boissons & Travailleurs exposés à la viande crue (78) \\
\hline & & Bâtiment et travaux publics & Travailleurs de la construction (79) \\
\hline & & $\begin{array}{l}\text { Autres services commerciaux et } \\
\text { personnels }\end{array}$ & Employés des restaurants (80) \\
\hline \multicolumn{4}{|c|}{ Zoonoses non entériques à transmission par vecteurs } \\
\hline $\begin{array}{l}\text { Encéphalite équine } \\
\text { de l'Est }\end{array}$ & $\begin{array}{l}\text { Oiseaux sauvages (e.g. } \\
\text { les passereaux) }\end{array}$ & Agriculture & Techniciens vétérinaires (81) \\
\hline \multirow{3}{*}{ Maladie de Lyme } & \multirow{3}{*}{$\begin{array}{l}\text { Souris à pattes blanches } \\
\text { (Peromyscus leucopus) }\end{array}$} & Agriculture & Travailleurs agricoles (82-85) \\
\hline & & Forets et scieries & Travailleurs forestiers (85) \\
\hline & & Administration publique & Personnel militaire (86-89) \\
\hline \multirow{3}{*}{$\begin{array}{l}\text { Virus du Nil } \\
\text { occidental }\end{array}$} & \multirow{3}{*}{$\begin{array}{l}\text { Oiseaux (surtout les } \\
\text { passereaux) }\end{array}$} & Services médicaux et sociaux & Personnel des laboratoires (90) \\
\hline & & $\begin{array}{l}\text { Autres services commerciaux et } \\
\text { personnels }\end{array}$ & Agents de contrôle des animaux (91) \\
\hline & & Agriculture & Étudiants en médecine vétérinaire (92) \\
\hline \multicolumn{4}{|c|}{ Zoonoses non entériques non transmises par des vecteurs } \\
\hline $\begin{array}{l}\text { Botulisme d'origine } \\
\text { alimentaire au } \\
\text { Nunavik }\end{array}$ & Phoques & Aucune information & Aucune information \\
\hline \multirow{5}{*}{ Fièvre Q } & \multirow{5}{*}{ Ruminants domestiques } & Administration publique & Personnel militaire (93-97) \\
\hline & & Agriculture & Travailleurs agricoles $(98,99)$ \\
\hline & & Industrie des aliments et boissons & Travailleurs dans les abattoirs (100) \\
\hline & & Industrie chimique & Travailleurs de l'industrie cosmétique $(101,102)$ \\
\hline & & Transport et entreposage & Conducteurs (103) \\
\hline $\begin{array}{l}\text { Grippe aviaire et } \\
\text { porcine }\end{array}$ & $\begin{array}{l}\text { Oiseaux (sauvages), } \\
\text { porcs }\end{array}$ & Agriculture & Travailleurs dans les élevages commerciaux de volailles (104) \\
\hline \multirow{3}{*}{ Rage } & \multirow{3}{*}{$\begin{array}{l}\text { Renards arctiques, } \\
\text { ratons laveurs, chauves- } \\
\text { souris }\end{array}$} & Administration publique & Personnel militaire $(105,106)$ \\
\hline & & Agriculture & Services vétérinaires (107) \\
\hline & & $\begin{array}{l}\text { Autres services commerciaux et } \\
\text { personnels }\end{array}$ & Employés en contact avec les chauves-souris (108) \\
\hline \multirow{4}{*}{$\begin{array}{l}\text { Syndrome } \\
\text { pulmonaire à } \\
\text { hantavirus }\end{array}$} & \multirow{4}{*}{$\begin{array}{l}\text { Souris sylvestre } \\
\text { (Peromyscus } \\
\text { maniculatus) }\end{array}$} & Agriculture & Travailleurs agricoles (109-111) \\
\hline & & Forêt et scieries & Travailleurs forestiers (109) \\
\hline & & Administration publique & Personnel militaire (112) \\
\hline & & $\begin{array}{l}\text { Autres services commerciaux et } \\
\text { personnels }\end{array}$ & $\begin{array}{l}\text { Piégeage et manipulation des rongeurs pour des études } \\
\text { écologiques (113) } \\
\text { Communications, transmission d'électricité et autres services } \\
\text { publics (114) }\end{array}$ \\
\hline
\end{tabular}




\section{Tableau 4 : Nombre d'articles retenus par zoonose prioritaire}

\begin{tabular}{|c|c|}
\hline Zoonoses prioritaires & $\begin{array}{l}\text { Nombre de publications } \\
\text { scientifiques pour lesquelles les } \\
\text { descriptions de cas satisfont } \\
\text { les critères des définitions } \\
\text { nosologiques et des critères } \\
\text { de diagnostic }\end{array}$ \\
\hline $\begin{array}{l}\text { Botulisme d'origine } \\
\text { alimentaire au Nunavik }\end{array}$ & 0 \\
\hline Campylobactériose & 16 \\
\hline Cryptosporidiose & 21 \\
\hline Encéphalite équine de l'Est & 1 \\
\hline $\begin{array}{l}\text { Escherichia coli à souche } \\
\text { verocytotoxigénique }\end{array}$ & 14 \\
\hline Fièvre Q & 11 \\
\hline Giardiase & 3 \\
\hline $\begin{array}{l}\text { Syndrome pulmonaire à } \\
\text { hantavirus }\end{array}$ & 6 \\
\hline Grippe aviaire et porcine & 1 \\
\hline Listériose & 3 \\
\hline Maladie de Lyme & 8 \\
\hline Rage & 4 \\
\hline Salmonellose & 20 \\
\hline Virus du Nil occidental & 3 \\
\hline Deux zoonoses ou plus & $2^{\mathrm{a}}$ \\
\hline Total & 111 \\
\hline
\end{tabular}

a Ces deux articles font partie de ceux qui ont été sélectionnés pour l'examen des connaissances sur les zoonoses visées, à savoir la campylobactériose, la cryptosporidiose et la salmonellose, mais ne sont comptés qu'une fois

Selon la littérature scientifique évaluée, les zoonoses les plus fréquemment signalées sur les lieux de travail sont les zoonoses entériques, suivies par les zoonoses non entériques à transmission non vectorielle et les zoonoses à transmission vectorielle. La salmonellose et la cryptosporidiose sont les zoonoses entériques les plus fréquemment mentionnées dans les articles évalués. Parmi les zoonoses à transmission vectorielle, la maladie de Lyme est la plus documentée, tandis que très peu d'articles traitant des arboviroses chez les travailleurs, tels le virus du Nil occidental et l'encéphalite équine de l'Est, ont été trouvés. Parmi les zoonoses non entériques non vectorielles, la plupart des articles scientifiques sélectionnés portaient sur la fièvre $Q$.

L'agriculture, qui inclut les services vétérinaires, est le secteur où le plus de zoonoses d'importance peuvent être contractées. Le secteur des services de l'administration publique, qui comprend la sécurité nationale et la défense, a également été identifié comme étant à risque pour l'acquisition des trois catégories de zoonoses, à savoir les zoonoses entériques, les zoonoses non entériques à transmission vectorielle et les zoonoses non entériques à transmission non vectorielle. Le troisième secteur le plus souvent mentionné est celui des services médicaux et sociaux, qui inclut entre autres le personnel des services de garde, des laboratoires, des hôpitaux, des centres de soins de longue durée et des maisons de retraite. Ce secteur a été indentifié comme présentant un risque accru pour l'acquisition des zoonoses entériques telles cryptosporidiose, E. coli vérocytotoxinogène, giardiase et salmonellose, ainsi qu'une zoonose à transmission vectorielle (transmission accidentelle du virus du Nil occidental parmi le personnel de laboratoire).

\section{Discussion}

Cette étude visait à décrire les professions et les secteurs d'activité à risque pour l'acquisition de zoonoses d'importance pour la santé publique au Québec. Les risques de contracter l'une des 14 zoonoses jugées importantes pour la santé publique par l'Observatoire multipartite sur les zoonoses et l'adaptation au climat du Québec varient d'une profession à l'autre. Les ouvriers agricoles et les vétérinaires, ainsi que le personnel militaire et le personnel des services médicaux et sociaux figurent parmi les travailleurs les plus fréquemment identifiés dans la littérature.

Il existe relativement peu de littérature qui a dressé ce type de portrait pour orienter les mesures de prévention en santé au travail. Deux études publiées nous ont cependant permis de comparer certaines observations. Une revue systématique de la littérature scientifique (publiée entre 1999 et 2008, sans restriction géographique) par Haagsma et al. (115) a examiné les accidents du travail imputables à des maladies infectieuses. La deuxième étude a présenté l'ampleur des accidents du travail imputables aux maladies infectieuses déclarées aux États-Unis entre 2006 et 2015 (116). Su et al. (116) ont examiné 67 publications scientifiques évaluées par des pairs (publiées entre 2006 et 2016) en suivant la méthodologie utilisée par Haagsma et al. (115), avant de terminer cette recherche en évaluant 66 rapports sur des cas de maladies infectieuses acquises sur le lieu de travail fournis par le Center for Disease du National Institute for Occupational Safety and Health.

Dans notre étude, le personnel militaire a été identifié comme étant à risque pour l'acquisition de trois catégories de zoonoses (entériques, non entériques à transmission vectorielle et non entériques à transmission non vectorielle), en particulier lors des missions à l'étranger. Ni Su et al. (116) ni Haagsma et al. (115) n'ont examiné amplement l'aspect militaire, sauf en ce qui concerne le risque associé à la leishmaniose, une infection parasitaire que l'on ne retrouve pas au Canada. Plusieurs des études axées sur le personnel militaire ont été publiées après 2008, c'est-à-dire après la période couverte par les études réalisées par Haagsma et al. (115) et Su et al. (116), ce qui explique une partie de la différence notée dans les observations entre ces études et nos recherches. La présente 
étude a identifié plusieurs facteurs de risque pour l'acquisition de zoonoses par le personnel militaire, incluant être basé dans des zones endémiques; participer à des camps d'entraînement dans des zones boisées ou à proximité de celles-ci (maladie de Lyme) $(87,88)$; vivre dans des structures ou des granges abandonnées dans lesquelles les animaux se sont reproduits, et travailler dans des sites de déploiement où la poussière devient aéroportée en raison des turbulences causées par les hélicoptères (fièvre Q) $(93,94,96,97)$.

Tout comme dans les observations de Su et al. (116), nous avons remarqué que les zoonoses entériques d'étiologie bactérienne sont les zoonoses professionnelles les plus fréquemment vues parmi les zoonoses d'importance. Cette étude a également montré que trois secteurs sont particulièrement touchés par les zoonoses d'importance, soit l'agriculture, ce qui inclut les services vétérinaires, les services de l'administration publique, incluant la défense, et les services médicaux et sociaux. Ce portrait a été observé par Haagsma et al. (115) et par Su et al. (116) qui rapportent que les travailleurs du secteur de la santé et ceux en contact avec des animaux sont les plus à risque d'être infectés par une variété de pathogènes zoonotiques en raison de leur niveau d'exposition. Les travailleurs de la santé sont donc principalement exposés à des agents pathogènes transmissibles d'humain à humain (115). Ils peuvent s'infecter accidentellement lorsqu'ils se blessent ou se piquent avec des aiguilles, ainsi que par contact direct avec la peau ou indirectement par contact oral-fécal, souvent en lien avec l'hygiène des mains. Su et al. (116) expliquent que les travailleurs en contact avec des animaux, en particulier le bétail ou la volaille, sont exposés au risque de contracter des zoonoses. Haagsma et al. (115) a identifié les agriculteurs, les travailleurs dans les abattoirs, les travailleurs en santé animale, les vétérinaires, les chasseurs et les jardiniers comme ceux qui sont à risque de contracter des zoonoses à la suite d'un contact avec des animaux. Toutes ces professions ont été indiquées dans notre étude comme étant à risque.

\section{Forces et faiblesse}

La principale limite de cette étude réside dans les critères d'inclusion et d'exclusion utilisés lors du processus de sélection des articles. La sélection des seules études publiées dont la description des cas zoonotiques répond aux définitions nosologiques ou aux critères de diagnostic peut avoir entraîné l'exclusion d'études présentant des cas d'infection asymptomatique diagnostiqués en laboratoire. Malgré cette limitation, les conclusions de notre étude sont similaires à celles de deux autres analyses documentaires $(115,116)$. Cependant, les résultats de cette étude reflètent un biais de publication. À titre d'exemple, il n'est pas surprenant qu'un plus grand nombre d'articles sur la maladie de Lyme aient été obtenus comparativement aux deux autres zoonoses à transmission vectorielle à l'étude en raison de l'ampleur des recherches récentes portant sur cette maladie. Cela appelle donc une interprétation prudente de l'importance de la documentation sur chacune des zoonoses.

\section{Conclusion}

Cette étude a dressé un portrait des professions et des secteurs d'activité les plus à risque pour l'acquisition de zoonoses prioritaires au Québec. L'agriculture (y compris les travailleurs du secteur vétérinaire), le personnel de l'administration publique (en particulier le personnel militaire) et des services médicaux et sociaux ont été identifiés comme étant les secteurs les plus touchés par les zoonoses prioritaires. Le personnel militaire a également été jugé à risque de contracter les trois catégories de zoonoses, puisque plusieurs facteurs de risque ont été soulevés pour l'acquisition de zoonoses chez ces derniers.

Dans l'ensemble, les risques de contracter des zoonoses sur le lieu de travail n'ont pas été largement étudiés. Les études à venir pourraient notamment inclure la consultation de représentants sur différents lieux de travail et d'experts en zoonoses pour pouvoir tirer parti des observations présentées. Il serait également utile de déterminer les mesures mises en place pour protéger la main-d'œuvre contre les zoonoses. Cela permettrait en ultimement de déterminer les éventuelles lacunes et de mieux orienter les efforts d'adaptation de la santé publique dans le contexte des changements climatiques.

\section{Déclaration des auteurs}

A. A. P. - Concept, rédaction du projet original, révision de la rédaction, examen critique

L. M. D. - Concept, rédaction du projet original, révision de la rédaction, examen critique

S. B. - Révision de la rédaction et de l'examen critique

G. G. - Révision de l'écriture et de la révision critique

A. I. C. - Révision de l'écriture et de la révision critique

M. P. S. - Révision de la rédaction et de l'examen critique

A. S. - Révision de la rédaction et de la révision critique

J. S. - Révision de l'écriture et de la révision critique

K. T. - Révision de l'écriture et de la révision critique

F. T. - Révision de l'écriture et de la révision critique

\section{Intérêts concurrents}

Aucun à déclarer.

\section{Remerciements}

Les auteurs tiennent à remercier Mme M. Lacourse de la Direction de la valorisation scientifique, des communications et de la performance organisationnelle de l'Institut national de santé publique du Québec pour son appui au moment de définir la stratégie de recherche bibliographique, ainsi que les membres du Comité de suivi pour leur importante contribution. 


\section{Financement}

Cette publication a été réalisée grâce à la participation financière obtenue dans le cadre du Plan d'action 2013-2020 sur les changements climatiques du gouvernement du Québec.

Le contenu de l'article et les points de vue qui y sont exprimés n'engagent que les auteurs et ne correspondent pas nécessairement à ceux du gouvernement du Canada.

\section{Références}

1. Germain G, Simon A, Arsenault J, Baron G, Bouchard C, Chaumont D, El Allaki F, Kimpton A, Lévesque B, Massé A, Mercier M, Ogden NH, Picard I, Ravel A, Rocheleau JP, Soto J. L'Observatoire multipartite québécois sur les zoonoses et l'adaptation aux changements climatiques. Relevé des maladies transmissibles au Canada 2019;45(5):159-64. DOI

2. Institut national de santé publique du Québec et Université de Montréal. Observatoire multi-partite québécois sur les zoonoses et l'adaptation aux changements climatiques. Bouchard C, Lowe AM, Simon A. Portrait des zoonoses priorisées par l'Observatoire multipartite québécois sur les zoonoses et l'adaptation aux changements climatiques en 2015. Montréal (QC) : INSPQ, 2017. https://www. inspq.qc.ca/sites/default/files/publications/2290_portrait_ zoonoses_priorisees_2015.pdf

3. Institut national de santé publique du Québec et Université de Montréal. Observatoire multi-partite québécois sur les zoonoses et l'adaptation aux changements climatiques. Simon A, Aenishaenslin C, Hongoh V, Lowe, AM. Priorisation des zoonoses au Québec dans un contexte d'adaptation aux changements climatiques à l'aide d'un outil d'aide à la décision mul-ticritère. Montréal (QC) : INSPQ, 2018. https:// www.inspq.qc.ca/sites/default/files/publications/2432 priorisation_zoonoses_quebec_outil_aide_decision_ multicritere.pdf

4. Campylobacter jejuni Infections Associated With Sheep Castration in Wyoming, 2011. MMWR 2011;60(48):1654.

5. de Perio MA, Niemeier RT, Levine SJ, Gruszynski K, Gibbins JD. Campylobacter infection in poultry-processing workers, Virginia, USA, 2008-2011. Emerg Infect Dis 2013;19(2):286-8. DOI PubMed

6. Ellis A, Irwin R, Hockin J, Borczyk A, Woodward D, Johnson W. Éclosion de cas d'infection à campylobacter chez des travailleurs agricoles; un exemple d'exposition en milieu de travail. Relevé des maladies transmissibles au Canada 1995;21(17):153-6. http://publications.gc.ca/ collections/Collection/H12-21-21-17F.pdf

7. Ellström P, Hansson I, Söderström C, Engvall EO, Rautelin H. A prospective follow-up study on transmission of Campylobacter from poultry to abattoir workers. Foodborne Pathog Dis 2014 Sep;11(9):684-8. DOI PubMed
8. Ganeshram KN, Ross A, Cowell RP, Cefai C, Woodward MJ. Recurring febrile illness in a slaughterhouse worker. Postgrad Med J 2000 Dec;76(902):790-1. DOI PubMed

9. Gilpin BJ, Scholes P, Robson B, Savill MG. The transmission of thermotolerant Campylobacter spp. to people living or working on dairy farms in New Zealand. Zoonoses Public Health 2008 Sep;55(7):352-60. DOI PubMed

10. Heryford AG, Seys SA. Outbreak of occupational campylobacteriosis associated with a pheasant farm. J Agric Saf Health 2004 May;10(2):127-32. DOI PubMed

11. Iraola G, Betancor L, Calleros L, Gadea P, Algorta G, Galeano S, Muxi P, Greif G, Pérez R. A rural worker infected with a bovine-prevalent genotype of Campylobacter fetus subsp. fetus supports zoonotic transmission and inconsistency of MLST and whole-genome typing. Eur J Clin Microbiol Infect Dis 2015;34(8):1593-6. DOI PubMed

12. Padungtod $P$, Kaneene JB. Campylobacter in food animals and humans in northern Thailand. J Food Prot 2005;68(12):2519-26. DOI PubMed

13. Wilson IG. Airborne Campylobacter infection in a poultry worker: case report and review of the literature. Commun Dis Public Health 2004;7(4):349-53. PubMed

14. Armed Forces Health Surveillance Center (AFHSC). Incidence of Campylobacter infections among service members of the active and reserve components of the U.S. Armed Forces and among other beneficiaries of the Military Health System, 2000-2013. MSMR 2014;21(12):11-6. PubMed

15. Beecham HJ 3rd, Lebron Cl, Echeverria P. Short report: impact of traveler's diarrhea on United States troops deployed to Thailand. Am J Trop Med Hyg 1997;57(6):699-701. DOl PubMed

16. Hennessy EP. An outbreak of campylobacteriosis amongst directing staff and students at the Infantry Training Centre, Brecon, Wales, March 2004. J R Army Med Corps 2004;150(3):175-8. DOI PubMed

17. O'Donnell FL, Stahlman S, Oh GT. Incidence of Campylobacter intestinal infections, active component, U.S. Armed Forces, 2007-2016. MSMR 2017;24(6):2-5. PubMed

18. Sanders JW, Isenbarger DW, Walz SE, Pang LW, Scott DA, Tamminga C, Oyofo BA, Hewitson WC, Sanchez JL, Pitarangsi C, Echeverria P, Tribble DR. An observational clinic-based study of diarrheal illness in deployed United States military personnel in Thailand: presentation and outcome of Campylobacter infection. Am J Trop Med Hyg 2002;67(5):533-8. DOI PubMed 
19. Tribble DR, Baqar S, Pang LW, Mason C, Houng HS, Pitarangsi C, Lebron C, Armstrong A, Sethabutr O, Sanders JW. Diagnostic approach to acute diarrheal illness in a military population on training exercises in Thailand, a region of campylobacter hyperendemicity. J Clin Microbiol 2008;46(4):1418-25. DOI PubMed

20. Benschop J, Booker CM, Shadbolt T, Weston JF. A Retrospective Cohort Study of an Outbreak of Cryptosporidiosis among Veterinary Students. Veterinary sciences. 2017;4(2). http://ovidsp.ovid.com/ovidweb.cgi?T=J $\mathrm{S} \& P A G E=$ reference $\& D=$ prem $\& N E W S=N \& A N=29056688$

21. Drinkard $L N$, Halbritter $A$, Nguyen GT, Sertich $P L$, King $M$, Bowman S, Huxta R, Guagenti M. Notes from the Field: Outbreak of Cryptosporidiosis Among Veterinary Medicine Students--Philadelphia, Pennsylvania, February 2015. MMWR Morb Mortal Wkly Rep 2015;64(28):773.

DOI PubMed

22. Gait R, Soutar RH, Hanson M, Fraser C, Chalmers R. Outbreak of cryptosporidiosis among veterinary students. Vet Rec 2008;162(26):843-5. DOI PubMed

23. Galuppi R, Piva S, Castagnetti C, Sarli G, lacono E, Fioravanti ML, Caffara M. Cryptosporidium parvum: from foal to veterinary students. Vet Parasitol 2016;219:53-6. DOI PubMed

24. Grinberg A, Pomroy WE, Squires RA, Scuffham A, Pita A, Kwan E. Retrospective cohort study of an outbreak of cryptosporidiosis caused by a rare Cryptosporidium parvum subgenotype. Epidemiol Infect 2011;139(10):1542-50. DOI PubMed

25. Kinross P, Beser J, Troell K, Axén C, Björkman C, Lebbad M, Winiecka-Krusnell J, Lindh J, Löfdahl M. Cryptosporidium parvum infections in a cohort of veterinary students in Sweden. Epidemiol Infect 2015;143(13):2748-56. DOI PubMed

26. Konkle DM, Nelson KM, Lunn DP. Nosocomial transmission of Cryptosporidium in a veterinary hospital. J Vet Intern Med 1997;11(6):340-3. DOl PubMed

27. Preiser G, Preiser L, Madeo L. An outbreak of cryptosporidiosis among veterinary science students who work with calves. J Am Coll Health 2003;51(5):213-5. DOI PubMed

28. Lassen B, Ståhl M, Enemark HL. Cryptosporidiosis - an occupational risk and a disregarded disease in Estonia. Acta Vet Scand 2014;56:36. DOI PubMed

29. El-Sherbini GT, Mohammad KA. Zoonotic cryptosporidiosis in man and animal in farms, Giza Governorate, Egypt. J Egypt Soc Parasitol 2006;36(2 Suppl):49-58. PubMed

30. Izadi M, Jonaidi-Jafari N, Saburi A, Eyni H, Rezaiemanesh MR, Ranjbar R. Cryptosporidiosis in Iranian Farm Workers and Their Household Members: A Hypothesis about Possible Zoonotic Transmission. J Trop Med 2014;2014:405875. DOI PubMed
31. Mahdi NK, Ali NH. Cryptosporidiosis among animal handlers and their livestock in Basrah, Iraq. East Afr Med J 2002;79(10):550-3. DOI PubMed

32. Ng JS, Eastwood K, Walker B, Durrheim DN, Massey PD, Porigneaux P, Kemp R, McKinnon B, Laurie K, Miller D, Bramley E, Ryan U. Evidence of Cryptosporidium transmission between cattle and humans in northern New South Wales. Exp Parasitol 2012;130(4):437-41. DOl PubMed

33. Siwila J, Phiri IG, Vercruysse J, Goma F, Gabriel S, Claerebout E, Geurden T. Asymptomatic cryptosporidiosis in Zambian dairy farm workers and their household members. Trans R Soc Trop Med Hyg 2007;101(7):733-4. DOI PubMed

34. Webb LM, Tubach SA, Hunt DC; Centers for Disease Control and Prevention (CDC). Outbreak of cryptosporidiosis among responders to a rollover of a truck carrying calves - Kansas, April 2013. MMWR Morb Mortal Wkly Rep 2014;63(50):1185-8. PubMed

35. Centers for Disease Control and Prevention (CDC). Outbreak of cryptosporidiosis associated with a firefighting response Indiana and Michigan, June 2011. MMWR Morb Mortal Wkly Rep 2012;61(9):153-6. PubMed

36. Centers for Disease Control and Prevention (CDC). Cryptosporidiosis outbreak at a summer camp-North Carolina, 2009. MMWR Morb Mortal Wkly Rep 2011;60(27):918-22. PubMed

37. Evans MR, Gardner D. Cryptosporidiosis outbreak associated with an educational farm holiday. CDR Rev 1996 Mar;6(3):R50-1. PubMed

38. Hoek MR, Oliver I, Barlow M, Heard L, Chalmers R, Paynter S. Outbreak of Cryptosporidium parvum among children after a school excursion to an adventure farm, south west England. J Water Health 2008;6(3):333-8. DOI PubMed

39. Cordell RL, Thor PM, Addiss DG, Theurer J, Lichterman R, Ziliak SR, Juranek DD, Davis JP. Impact of a massive waterborne cryptosporidiosis outbreak on child care facilities in metropolitan Milwaukee, Wisconsin. Pediatr Infect Dis J 1997;16(7):639-44. DOI PubMed

40. Hancock-Allen J, Alden NB, Cronquist AB. Cryptosporidiosis outbreak at an academic animal research laboratory-Colorado, 2014. Am J Ind Med 2017;60(2):208-14. DOI PubMed

41. Chalmers RM, Salmon RL, Willshaw GA, Cheasty T, Looker N, Davies I, Wray C. Vero-cytotoxin-producing Escherichia coli O157 in a farmer handling horses. Lancet 1997;349(9068):1816. DOI PubMed

42. Hong S, Oh KH, Cho SH, Kim JC, Park MS, Lim HS, Lee BK. Asymptomatic healthy slaughterhouse workers in South Korea carrying Shiga toxin-producing Escherichia coli. FEMS Immunol Med Microbiol 2009;56(1):41-7. DOI PubMed 
43. Hong S, Song SE, Oh KH, Kim SH, Yoo SJ, Lim HS, Park MS. Prevalence of Farm and Slaughterhouse Workers Carrying Shiga Toxin-Producing Escherichia coli in Korea. Osong Public Health Res Perspect 2011;2(3):198-201. DOI PubMed

44. Park JH, Lim HS, Lee K, Hong S, Park MS. Prevalence of Shiga toxin-encoding genes and risk factors among dairy farmers in Gyeonggi Province, Korea. Scand J Infect Dis 2011;43(4):275-9. DOI PubMed

45. Rehman MU, Mohd R, Maninder S, Nitasha S, Reshi IA. Isolation, characterization and association of Shiga toxin-producing Escherichia coli from bovines and their handlers in Jammu, India. J Pure Appl Microbiol 2014;8(3):2353-8. https://microbiologyjournal.org/ isolation-characterization-and-association-of-shig a-toxin-producing-escherichia-coli-from-bovines-an d-their-handlers-in-jammu-india/

46. Silvestro L, Caputo M, Blancato S, Decastelli L, Fioravanti A, Tozzoli R, Morabito S, Caprioli A. Asymptomatic carriage of verocytotoxin-producing Escherichia coli O157 in farm workers in Northern Italy. Epidemiol Infect 2004;132(5):915-9. DOI PubMed

47. Stephan R, Untermann F. Virulence factors and phenotypical traits of verotoxin-producing Escherichia coli strains isolated from asymptomatic human carriers. J Clin Microbiol 1999;37(5):1570-2. DOI PubMed

48. Durso LM, Reynolds K, Bauer N Jr, Keen JE. Shiga-toxigenic Escherichia coli O157:H7 infections among livestock exhibitors and visitors at a Texas County Fair. Vector Borne Zoonotic Dis 2005;5(2):193-201. DOI PubMed

49. Brown JA, Hite DS, Gillim-Ross LA, Maguire HF, Bennett JK, Patterson JJ, Comstock NA, Watkins AK, Ghosh TS, Vogt RL. Outbreak of shiga toxin-producing Escherichia coli serotype O26: $\mathrm{H} 11$ infection at a child care center in Colorado. Pediatr Infect Dis J 2012;31(4):379-83. DOI PubMed

50. O'Donnell JM, Thornton L, McNamara EB, Prendergast T, Igoe D, Cosgrove C. Outbreak of Vero cytotoxin-producing Escherichia coli $\mathrm{O} 157$ in a child day care facility. Commun Dis Public Health 2002;5(1):54-8. PubMed

51. Afza M, Hawker J, Thurston H, Gunn K, Orendi J. An outbreak of Escherichia coli $\mathrm{O} 157$ gastroenteritis in a care home for the elderly. Epidemiol Infect 2006;134(6):1276-81. DOI PubMed

52. Weightman NC, Kirby PJ. Nosocomial Escherichia coli $O 157$ infection. J Hosp Infect 2000;44(2):107-11. DOI PubMed

53. Bayliss L, Carr R, Edeghere O, Knapper E, Nye K, Harvey G, Adak G, Duggal H. School outbreak of Escherichia coli O157 with high levels of transmission, Staffordshire, England, February 2012. J Public Health (Oxf) 2016;38(3):e247-53. DOI PubMed
54. Porter CK, Riddle MS, Tribble DR, Putnam SD, Rockabrand DM, Frenck RW, Rozmajzl P, Kilbane E, Fox A, Ruck R, Lim M, Johnston J, Murphy E, Sanders JW. The epidemiology of travelers' diarrhea in Incirlik, Turkey: a region with a predominance of heat-stabile toxin producing enterotoxigenic Escherichia coli. Diagn Microbiol Infect Dis 2010;66(3):241-7. DOI PubMed

55. Ang LH. Outbreak of giardiasis in a daycare nursery. Commun Dis Public Health 2000;3(3):212-3. PubMed

56. Guimarães S, Sogayar MI. Occurrence of Giardia lamblia in children of municipal day-care centers from Botucatu, São Paulo State, Brazil. Rev Inst Med Trop São Paulo 1995;37(6):501-6. DOI PubMed

57. Linnane E, Roberts R, Looker N. Nappies and transmission of Giardia lamblia between children. Lancet 2001;358(9280):507. DOI PubMed

58. Laureyns J, Moyaert H, Werbrouck H, Catry B, de Kruif A, Pasmans F. Pustular dermatitis by Listeria monocytogenes after the assisted delivery of a dead calf. Vlaams Diergeneeskd Tijdschr 2008;77(1):29-34. https://vdt.ugent. be/sites/default/files/art77105.pdf

59. Regan EJ, Harrison GA, Butler S, McLauchlin J, Thomas M, Mitchell S. Primary cutaneous listeriosis in a veterinarian. Vet Rec 2005;157(7):207. DOI PubMed

60. Gilchrist M. Cutaneous Listeria infection. British journal of hospital medicine (London, England : 2005). 2009;70(11):659.

61. Lazarus R, Waghorn D, Nash C. Cutaneous Salmonella infection. Scand J Infect Dis 2007;39(3):257-8. DOI PubMed

62. Bemis DA, Craig LE, Dunn JR. Salmonella transmission through splash exposure during a bovine necropsy. Foodborne Pathog Dis 2007;4(3):387-90. DOI PubMed

63. Anonymous. From the Centers for Disease Control and Prevention. Outbreaks of multidrug-resistant Salmonella typhimurium associated with veterinary facilities-Idaho, Minnesota, and Washington, 1999. JAMA 2001;286(16):1965-6. PubMed

64. Cherry B, Burns A, Johnson GS, Pfeiffer H, Dumas N, Barrett D, McDonough PL, Eidson M. Salmonella Typhimurium outbreak associated with veterinary clinic. Emerg Infect Dis 2004;10(12):2249-51. DOI PubMed

65. Padungtod P, Kaneene JB. Salmonella in food animals and humans in northern Thailand. Int J Food Microbiol 2006;108(3):346-54. DOI PubMed

66. Gong J, Wang C, Shi S, Bao H, Zhu C, Kelly P, Zhuang L, Lu G, Dou X, Wang R, Xu B, Zou J. Highly Drug-Resistant Salmonella enterica Serovar Indiana Clinical Isolates Recovered from Broilers and Poultry Workers with Diarrhea in China. Antimicrob Agents Chemother 2016;60(3):1943-7. DOI PubMed 
67. Baker MG, Thornley CN, Lopez LD, Garrett NK, Nicol CM. A recurring salmonellosis epidemic in New Zealand linked to contact with sheep. Epidemiol Infect 2007;135(1):76-83. DOI PubMed

68. Prapasarakul N, Pulsrikarn C, Vasaruchapong T, Lekcharoen P, Chanchaithong P, Lugsomya K, Keschumras N, Thanomsuksinchai N, Tanchiangsai K, Tummaruk P. Salmonella serovar distribution in cobras (Naja kaouthia), snake-food species, and farm workers at Queen Saovabha Snake Park, Thailand. Journal of veterinary diagnostic investigation Inc. 2012;24(2):288-94. DOI

69. Alexander DC, Fitzgerald SF, DePaulo R, Kitzul R, Daku D, Levett PN, Cameron AD. Laboratory-Acquired Infection with Salmonella enterica Serovar Typhimurium Exposed by Whole-Genome Sequencing. J Clin Microbiol 2016;54(1):190-3. DOI PubMed

70. Centers for Disease Control and Prevention (CDC). Occupationally acquired Salmonella I 4,12:i:1,2 infection in a phlebotomist--Minnesota, January 2013. MMWR Morb Mortal Wkly Rep 2013;62(25):525. PubMed

71. Centers for Disease Control and Prevention (CDC). Salmonella serotype enteritidis infections among workers producing poultry vaccine--Maine, November-December 2006. MMWR Morb Mortal Wkly Rep 2007;56(34):877-9. PubMed

72. Standaert SM, Hutcheson RH, Schaffner W. Nosocomial transmission of Salmonella gastroenteritis to laundry workers in a nursing home. Infect Control Hosp Epidemiol 1994;15(1):22-6. DOI PubMed

73. Newcomb S, Broadhurst L, Kissane K. Salmonella outbreak in an American child development center in Germany. Mil Med 1997;162(12):783-7. DOl PubMed

74. Dontsenko I, Võzelevskaja N, Põld A, Kerbo N, Kutsar K. Outbreak of salmonellosis in a kindergarten in Estonia, May 2008. Euro Surveill 2008;13(24):18900. PubMed

75. Clark LL, Daniele DO, O’Donnell FL. Incidence of Salmonella infections among service members of the active and reserve components of the U.S. Armed Forces and among other beneficiaries of the Military Health System, 2000-2013. MSMR 2015;22(1):11-5. PubMed

76. Williams VF, Stahlman S, Oh GT. Incidence of nontyphoidal Salmonella intestinal infections, active component, U.S. Armed Forces, 2007-2016. MSMR 2017;24(6):6-10. PubMed

77. Bartholomew ML, Heffernan RT, Wright JG, Klos RF, Monson T, Khan S, Trees E, Sabol A, Willems RA, Flynn R, Deasy MP, Jones B, Davis JP. Multistate outbreak of Salmonella enterica serotype enteritidis infection associated with pet guinea pigs. Vector Borne Zoonotic Dis 2014;14(6):414-21. DOI PubMed
78. Doorduyn $Y$, Van Den Brandhof WE, Van Duynhoven YT, Wannet WJ, Van Pelt W. Risk factors for Salmonella Enteritidis and Typhimurium (DT104 and non-DT104) infections in The Netherlands: predominant roles for raw eggs in Enteritidis and sandboxes in Typhimurium infections. Epidemiol Infect 2006;134(3):617-26. DOI PubMed

79. Faustini A, Sangalli M, Fantasia M, Manganello R, Mattaccini E, Trippanera R, Spera D, La Rosa U, Topi MT, Forastiere F, Perucci CA. An outbreak of Salmonella hadar associated with food consumption at a building site canteen. Eur J Epidemiol 1998;14(1):99-106. DOI PubMed

80. Evans MR, Hutchings PG, Ribeiro CD, Westmoreland D. A hospital outbreak of salmonella food poisoning due to inadequate deep-fat frying. Epidemiol Infect 1996;116(2):155-60. DOI PubMed

81. Min Z, Gnann JW Jr. Mosquito bites and eastern equine encephalitis. QJM International Journal of Medicine. 2014;107(5):397-8. DOI PubMed

82. Stawicki T. Analysis of the variability of registered cases of Lyme disease among workers of private agriculture in West Pomeranian Voivodship. Folia Pomeranae Universitatis Technologiae Stetinensis, Agricultura, Alimentaria, Piscaria et Zootechnica. 2017;334(42/2):175-85. http://foliaaapz.zut. edu.pl/pdf/files/magazines/1/69/936.pdf

83. Zając V, Pinkas J, Wójcik-Fatla A, Dutkiewicz J, Owoc A, Bojar I. Prevalence of serological response to Borrelia burgdorferi in farmers from eastern and central Poland. Eur $\mathrm{J}$ Clin Microbiol Infect Dis 2017;36(3):437-46. DOI PubMed

84. Szeszenia-Dąbrowska N, Świątkowska B, Wilczńska U. Occupational diseases among farmers in Poland. Med $\mathrm{Pr}$ 2016;67(2):163-71. DOI PubMed

85. Bilski B. Occurrence of cases of borreliosis certified as an occupational disease in the province of Wielkopolska (Poland). Ann Agric Environ Med 2009;16(2):211-7. PubMed

86. Anna MM, Escobar JD, Chapman AS. Reported vectorborne and zoonotic diseases, U.S. Air Force, 2000-2011. MSMR 2012;19(10):11-2. PubMed

87. Hurt L, Dorsey KA. The geographic distribution of incident Lyme disease among active component service members stationed in the continental United States, 2004-2013. MSMR 2014;21(5):13-5. PubMed

88. Rossi C, Stromdahl EY, Rohrbeck P, Olsen C, DeFraites RF. Characterizing the relationship between tick bites and Lyme disease in active component U.S. Armed Forces in the eastern United States. MSMR 2015;22(3):2-10. PubMed

89. Armed Forces Health Surveillance Center (AFHSC). Surveillance snapshot: lyme disease among beneficiaries of the Military Health System, 2001-2012. MSMR 2013;20(8):23. PubMed 
90. Centers for Disease Control and Prevention (CDC). Laboratory-acquired West Nile virus infections-United States, 2002. MMWR Morb Mortal Wkly Rep 2002;51(50):1133-5. PubMed

91. Fonseca K, Prince GD, Bratvold J, Fox JD, Pybus M, Preksaitis JK, Tilley P. West Nile virus infection and conjunctival exposure. Emerg Infect Dis 2005;11(10):1648-9. DOI PubMed

92. Venter M, Steyl J, Human S, Weyer J, Zaayman D, Blumberg L, Leman PA, Paweska J, Swanepoel R. Transmission of West Nile virus during horse autopsy. Emerg Infect Dis 2010;16(3):573-5. DOI PubMed

93. Anderson AD, Baker TR, Littrell AC, Mott RL, Niebuhr DW, Smoak BL. Seroepidemiologic survey for Coxiella burnetii among hospitalized US troops deployed to Iraq. Zoonoses Public Health 2011;58(4):276-83. DOI PubMed

94. Faix DJ, Harrison DJ, Riddle MS, Vaughn AF, Yingst SL, Earhart K, Thibault G. Outbreak of $Q$ fever among US military in western Iraq, June-July 2005. Clin Infect Dis 2008;46(7):e65-8. DOl PubMed

95. Hartzell JD, Peng SW, Wood-Morris RN, Sarmiento DM, Collen JF, Robben PM, Moran KA. Atypical Q fever in US soldiers. Emerg Infect Dis 2007;13(8):1247-9. DOI PubMed

96. Royal J, Riddle MS, Mohareb E, Monteville MR, Porter CK, Faix DJ. Seroepidemiologic survey for Coxiella burnetii among US military personnel deployed to Southwest and Central Asia in 2005. Am J Trop Med Hyg 2013;89(5):991-5. DOI PubMed

97. White B, Brooks T, Seaton RA. Q fever in military and paramilitary personnel in conflict zones: case report and review. Travel Med Infect Dis 2013;11(2):134-7. DOI PubMed

98. Towey A, Petitti C. OSHA Compliance Issues. J Occup Environ Hyg 2009;6(10):63-5. DOI PubMed

99. Hatchette T, Hudson R, Schlech W, Campbell N, Hatchette $\mathrm{J}$, Ratnam S, Marrie T. Fièvre q associée à des caprins à Terre-Neuve. Relevé des maladies transmissibles au Canada 2000;26(3):17-9. http://publications.gc.ca/collections/ Collection/H12-21-26-3.pdf

100. Aw TC, Ratti N. Occupational infection in an offal porter: a case of $Q$ fever. Occup Med (Lond) 1997;47(7):432-4. DOI PubMed

101.Wade AJ, Cheng AC, Athan E, Molloy JL, Harris OC, Stenos J, Hughes AJ. Q fever outbreak at a cosmetics supply factory. Clin Infect Dis 2006;42(7):e50-2. DOI PubMed

102. Wade AJ, Walker T, Athan E, Hughes AJ. Spontaneous splenic rupture: a rare complication of $\mathrm{Q}$ fever in Australia. Med J Aust 2006;184(7):364. DOI PubMed

103. Milazzo A, Hall R, Storm PA, Harris RJ, Winslow W, Marmion BP. Sexually transmitted $Q$ fever. Clin Infect Dis 2001;33(3):399-402. DOl PubMed
104. Koopmans M, Wilbrink B, Conyn M, Natrop G, van der Nat $H$, Vennema $H$, Meijer A, van Steenbergen J, Fouchier R, Osterhaus A, Bosman A. Transmission of H7N7 avian influenza $A$ virus to human beings during a large outbreak in commercial poultry farms in the Netherlands. Lancet 2004;363(9409):587-93. DOI PubMed

105. Cooper ED, Debboun M. The relevance of rabies to today's military. US Army Med Dep J 2012 Jul-Sep;4-11. PubMed

106. Centers for Disease Control and Prevention (CDC). Imported human rabies in a U.S. Army soldier - New York, 2011. MMWR Morb Mortal Wkly Rep 2012;61(17):302-5. PubMed

107. Brito MG, Chamone TL, da Silva FJ, Wada MY, Miranda AB, Castilho JG, Carrieri ML, Kotait I, Lemos FL. Antemortem diagnosis of human rabies in a veterinarian infected when handling a herbivore in Minas Gerais, Brazil. Rev Inst Med Trop São Paulo 2011;53(1):39-44. DOl PubMed

108. Powell JC. Evaluating risk: rabies exposure and occupational implications. AAOHN journal 2009;57(11):465-463. DOI PubMed

109. Castillo C, Naranjo J, Sepúlveda A, Ossa G, Levy H. Hantavirus pulmonary syndrome due to Andes virus in Temuco, Chile: clinical experience with 16 adults. Chest 2001;120(2):548-54. DOl PubMed

110. Cruz CD, Forshey BM, Vallejo E, Agudo R, Vargas J, Blazes DL, Guevara C, Laguna-Torres VA, Halsey ES, Kochel TJ. Novel strain of Andes virus associated with fatal human infection, central Bolivia. Emerg Infect Dis 2012;18(5):750-7. DOI PubMed

111. Martinez VP, Bellomo CM, Cacace ML, Suarez $P$, Bogni $L$, Padula PJ. Hantavirus pulmonary syndrome in Argentina, 1995-2008. Emerg Infect Dis 2010;16(12):1853-60. DOl PubMed

112. Parkes LO, Nguyen TT, Longtin J, Beaudoin MC, Bestman-Smith J, Vinh DC, Boivin G, Loo VG. A Cluster of Three Cases of Hantavirus Pulmonary Syndrome among Canadian Military Personnel. Can J Infect Dis Med Microbiol 2016;2016:2757969. DOI PubMed

113. Torres-Perez F, Wilson L, Collinge SK, Harmon H, Ray C, Medina RA, Hjelle B. Sin Nombre virus infection in field workers, Colorado, USA. Emerg Infect Dis 2010;16(2):308-10. DOI PubMed

114.Jay M, Hjelle B, Davis R, Ascher M, Baylies HN, Reilly K, Vugia D. Occupational exposure leading to hantavirus pulmonary syndrome in a utility company employee. Clin Infect Dis 1996;22(5):841-4. DOI PubMed

115. Haagsma JA, Tariq L, Heederik DJ, Havelaar AH. Infectious disease risks associated with occupational exposure: a systematic review of the literature. Occup Environ Med 2012;69(2):140-6. DOI PubMed

116.Su CP, de Perio MA, Cummings KJ, McCague AB, Luckhaupt SE, Sweeney MH. Case Investigations of Infectious Diseases Occurring in Workplaces, United States, 2006-2015. Emerg Infect Dis 2019;25(3):397-405. DOI PubMed 